\title{
Individual differences in FFA activity suggest independent processing at different spatial scales
}

\author{
ISABEL GAUTHIER and KIM M. CURBY \\ Vanderbilt University, Nashville, Tennessee \\ PAWEL SKUDLARSKI \\ Yale School of Medicine, New Haven, Connecticut \\ and \\ RUSSELL A. EPSTEIN \\ University of Pennsylvania, Philadelphia, Pennsylvania
}

\begin{abstract}
The brain processes images at different spatial scales, but it is unclear how far into the visual stream different scales remain segregated. Using functional magnetic resonance imaging, we found evidence that BOLD activity in the fusiform face area (FFA) reflects computations based on separate spatial frequency inputs. When subjects perform different tasks (attend location vs. identity; attend whole vs. parts) or the same task with different stimuli (upright or inverted) with high- and low-pass images of cars and faces, individual differences in the FFA in one condition are correlated with those in the other condition. However, FFA activity in response to low-pass stimuli is independent of its response to highpass stimuli. These results suggest that spatial scales are not integrated before the FFA and that processing in this area could support the flexible use of different sources of information present in broadpass images.
\end{abstract}

We can process visual information over a wide range of spatial scales, perceiving both the stripes on the zebra and the herd moving as a whole within the same glance. This ability to detect contrast at several spatial scales is mediated by a number of narrowly tuned mechanisms, as has been shown by behavioral experiments demonstrating masking or adaptation selective to only one of these putative channels (Blakemore \& Campbell, 1969; Campbell \& Robson, 1968; Gilinsky, 1968; Legge \& Foley, 1980). Such channels arise as a result of the selectivity of neurons in the early visual cortex to different spatial frequencies (Blakemore \& Campbell, 1969; Movshon, Thompson, \& Tolhurst, 1978; Robson, Tolhurst, Freeman, \& Ohzawa, 1988). Although there has been a debate concerning whether visual perception operates independently at each spatial scale or on features combining different spatial frequencies, recent work has revealed both within-scale and combined-scale effects, depending on the task (see Hess, 2004, for a review).

This work was supported by the James S. McDonnell Foundation, the National Eye Institute, and the National Science Foundation (to I.G.). We thank Aude Oliva for providing image filters, Adam W. Anderson for help with programming and useful discussions, Andy Tomarken for statistical advice, and the members of the Perceptual Expertise Network (funded by the JSMF), René Marois, and Thomas W. James for comments on earlier versions of the manuscript. Correspondence concerning this article should be addressed to I. Gauthier, Psychology Department, Vanderbilt University, 301 Wilson Hall, Nashville, TN 37203 (e-mail: isabel.gauthier@vanderbilt.edu).
Face recognition, for instance, appears to rely on features that combine information across different scales (Schyns, Bonnar, \& Gosselin, 2002). However, behavioral studies often can reveal only the end product of several complex stages of processing, offering little evidence as to how and where in the brain information at different scales is combined. How far along the processing stream does coarse and fine spatial information remain segregated? Is the processing of category-selective areas in the ventral occipito-temporal cortex invariant to spatial scales? Here, we focus on the fusiform face area (FFA), a cortical area that is involved in face recognition (Kanwisher, McDermott, \& Chun, 1997; Sergent, Ohta, \& MacDonald, 1992) and has also been related to expertise level with nonface object recognition (Gauthier, Skudlarski, Gore, \& Anderson, 2000; Gauthier, Tarr, Anderson, Skudlarski, \& Gore, 1999). We ask whether the coarse and the fine information about a face or an object of expertise converges in representations of single individuals at this level of cortical processing. To fully understand the nature of the computations taking place in a cortical area, it is important to characterize the level of abstraction at which it represents objects.

However, there is conflicting evidence on whether FFA activity is spatial frequency invariant or not. On the one hand, a recent fMRI study that used a priming paradigm in which observers made gender decisions showed that activation in a region of the fusiform gyrus was affected by repetition of personal identity, regardless of changing spatial scale (Eger, Schyns, \& Kleinschmidt, 
2004). In addition, face cells in the macaque monkey can respond to different face images separated by three octaves in spatial frequency content (Rolls, Baylis, \& Leonard, 1985). It is possible that face representations in the fusiform gyrus are invariant to spatial scale, just as they appear to be size and location invariant (Grill-Spector et al., 1999).

On the other hand, other results argue against spatial frequency invariant representations in the FFA. Face recognition and face matching are strongly influenced by spatial frequency overlap between images (the more overlap in spatial frequency content, the easier it is to match two faces), supporting the idea that face judgments may depend on an array of spatial frequency channels (Liu, Collin, Rainville, \& Chaudhuri, 2000). And although neurophysiological results do suggest that face cells respond over a wide range of spatial frequencies, the response to very low pass (low spatial frequency, LSF) and high pass (high spatial frequency, HSF) images (e.g., $<8$ cycles/image [c/i] and $>32 \mathrm{c} / \mathrm{i}$ ) is such that some neurons show an LSF preference, whereas others show an HSF preference (Rolls et al., 1985). In addition, in this experiment, neurons that tended to respond well to images of face parts presented alone also showed a preference for HSFs. Of course, these neurophysiological results may or may not relate directly to response patterns in the human FFA: Homologies between faceselective areas in the human and the monkey remain to be established (Tsao, Freiwald, Knutsen, Mandeville, \& Tootell, 2003). But there is also human fMRI data suggesting spatial scale effects in the FFA. One fMRI study (Vuilleumier, Armony, Driver, \& Dolan, 2003) in which the effect of face identity repetition across different spatial scales was investigated and in which observers also made gender decisions (as in Eger et al., 2004) showed a surprising asymmetrical habituation effect in the fusiform gyrus. Specifically, habituation was obtained when a face was first seen in an HSF version and later in an LSF version, but not the other way around (Vuilleumier et al., 2003). This is inconsistent with the existence of face representations invariant to spatial scale, which should lead to a symmetrical effect. Indeed, the demonstration of asymmetrical habituation effects suggests that habituation (even if the effects are symmetrical) can occur even if the two stimuli do not fully overlap in their representation. Habituation effects can also be strategic. For instance, priming paradigms can be complicated by interactions with stimulus familiarity, possibly mediated by naming of familiar stimuli (Henson, Shallice, \& Dolan, 2000). In addition, the longer the exposure to a degraded image, the more interference coming from perceptual guesses can hinder recognition of a subsequent image of the same object (Bruner \& Potter, 1964). This is more likely to play a role in studies in which different filtered versions of the same face are presented back to back.

Main effects of spatial frequency content have been reported in the fusiform cortex, but on the other hand, there is also evidence that the face-selective responses in the FFA do not depend on spatial frequency content. Sasaki et al. (2001) found that spatial frequency sensitivity covaries with the retinotopic representation of eccentricity, with higher frequencies near the foveal representation. In addition, Malach and colleagues (Hasson, Harel, Levy, \& Malach, 2003; Levy, Hasson, Avidan, Hendler, \& Malach, 2001) have shown that the FFA is in a center-biased region of the cortex. This suggests that the FFA could show a preference for HSF, and indeed, HSF faces ( $>24 \mathrm{cpi}$ ) lead to more activity in the fusiform and the inferior temporal gyrus than LSF faces do $(<6 \mathrm{cpi}$; Eger et al., 2004; Winston, Vuilleumier, \& Dolan, 2003). However, activity in the FFA itself appears to be relatively insensitive to spatial frequency content (Lerner, Hendler, Ben-Bashat, Harel, \& Malach, 2001; Malach et al., 1995), in accord with reports that the selectivity for faces over objects that defines this area (Haxby et al., 1999; Kanwisher et al., 1997; McCarthy, Puce, Gore, \& Allison, 1997; Puce, Allison, Gore, \& McCarthy, 1995; Sergent et al., 1992) can be obtained as well with grayscale images, two-tone images, or line-drawings (Ishai, Ungerleider, Martin, Schouten, \& Haxby, 1999; Kanwisher, Tong, \& Nakayama, 1998). Intracranial recordings also reveal that early face-sensitive responses in the fusiform gyrus are found for both blurred and line-drawn faces (although line-drawn faces led to responses delayed by about $30 \mathrm{msec}$, as compared with normal images; McCarthy, Puce, Belger, \& Allison, 1999). Therefore, despite overall higher responses to HSF images in the fusiform gyrus, selective responses in the FFA can be obtained for images containing either only HSF or only LSF information.

Finding category-selective responses in the FFA for images containing either only HSF or only LSF could be explained in at least two different ways: Cells in this area could all be invariant to spatial scale, or there could be different spatial frequency channels intermixed within each voxel of this small region, resulting in a lack of an overall spatial frequency effect (Figure 1). Note that these are extreme hypotheses about spatial frequency channels in the FFA: The limited neurophysiological evidence on the effect of spatial scale in monkey superior temporal sulcus face cells suggests a more complex picture. Although individual cells can respond to a range of spatial frequencies, sometimes including both HSFs and LSFs, different cells show different scale preferences for highly filtered images, and the selectivity of these neurons appears to be a combination of excitation and inhibition at different scales (Rolls et al., 1985). Here, we are concerned with the functional independence of different spatial scales in the neural signals mediating selective responses in the human FFA, so we begin this investigation with a cartoon assumption about the independence of possible spatial channels within the same voxel.

These very different hypotheses about the organization of spatial scales at the level of the FFA cannot be distinguished simply by looking at the mean activity elicited in different spatial frequency conditions. We 
A

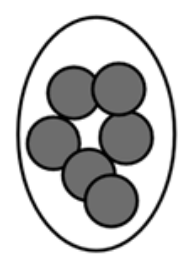

C

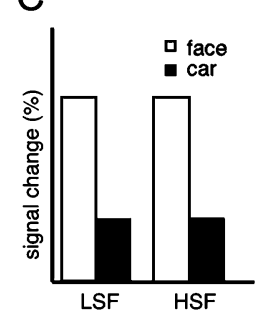

$E$

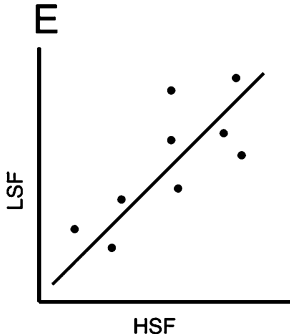

G

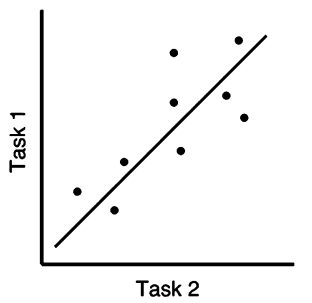

$\mathrm{B}$

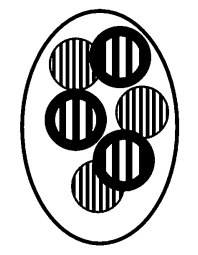

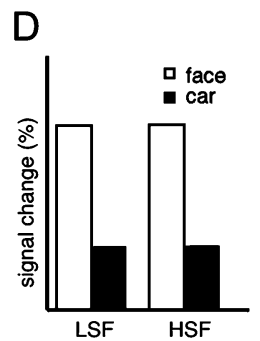
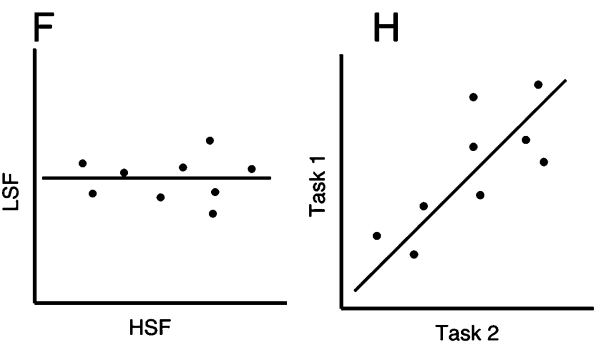

Figure 1. Schematic representation of the predictions. The fusiform face area (FFA) could contain cells that respond regardless of spatial frequency content $(A)$ or cells that are tuned to different bands of spatial frequencies (B). Because of the limited spatial resolution of $f$ MRI, the average signal in the FFA for faces and cars shown in low spatial frequency (LSF) or high spatial frequency (HSF) format would yield the same pattern (C and D), regardless of the organization within the FFA. In contrast, the correlation of individual differences for the responses in the FFA to LSF versus HSF images has the potential to disambiguate the difference between $A$ and $B$. If there is just one population of cells whose response is invariant to spatial scale, it should mediate the FFA response, regardless of the spatial frequency content in the stimuli, and individual differences should show a strong correlation across this manipulation $(E)$. Note that correlated individual differences for spatial frequencies (as in $\mathbf{E}$ ) would be an ambiguous finding, because different populations of neurons could lead to this pattern if their responses were "yoked" in some way. However, if no such correlation is obtained (F), this suggests that different neuronal populations exist, as in B, and that the relative size (and/or activity) of these populations across individuals is independent. Any convergence of inputs at any point in the visual-processing stream before the FFA should lead to a correlation of individual differences in FFA activity across spatial scales. In our factorial design, a task manipulation orthogonal to the spatial frequency manipulation should lead to a strong correlation, regardless of the organization within the FFA ( $G$ and $H$ ), as long as the same neurons contribute to both tasks. This relationship provides a basis with which the correlation across spatial frequency can be compared. It also controls for the possibility that independence (as in F) could be attributed to noise or other artifacts in the data.

present an alternative approach focusing on the correlation of individual differences in fMRI activity across different conditions (see Figure 1). We assume that, as the literature suggests, FFA activity for a group of subjects will show comparable selectivity for images containing LSF or HSF. If this is the case, and if selectivity for HSF and LSF comes from the same spatial frequency invariant neurons, subjects who have a strong FFA selectivity with HSF images should also have strong FFA selectivity with LSF images. In contrast, if individual differences in FFA selectivity for LSF and HSF are not correlated, this would indicate that they arise from different neuronal populations. Because the absence of a correlation is a null effect, our experiments compared a spatial frequency manipulation with other task manipulations, to test the hypothesis that individual differences correlate across these conditions more than across changes in spatial scale.
In three experiments, we used three-way factorial designs (see Figure 2), manipulating the spatial frequency content of the images (HSF or LSF) and either the task required of the observers (Experiment 1, attention to wholes vs. parts; Experiment 2, attention to identity vs. location) or the orientation of the stimuli (Experiment 3, upright vs. inverted). In each experiment, we also manipulated the object category, using cars and faces. Faces are the stimuli of choice to elicit a maximal response in the FFA; objects such as cars should lead to a much smaller response in this region (Kanwisher et al., 1997), except in subjects who are car experts (Gauthier, Skudlarski, et al., 2000). Because our analyses focused on correlations across subjects, variations in car expertise provided a convenient way to maximize individual differences in FFA activity. One limitation of prior fMRI studies with spatially filtered faces is that face-selective areas in the fusiform were not defined functionally 

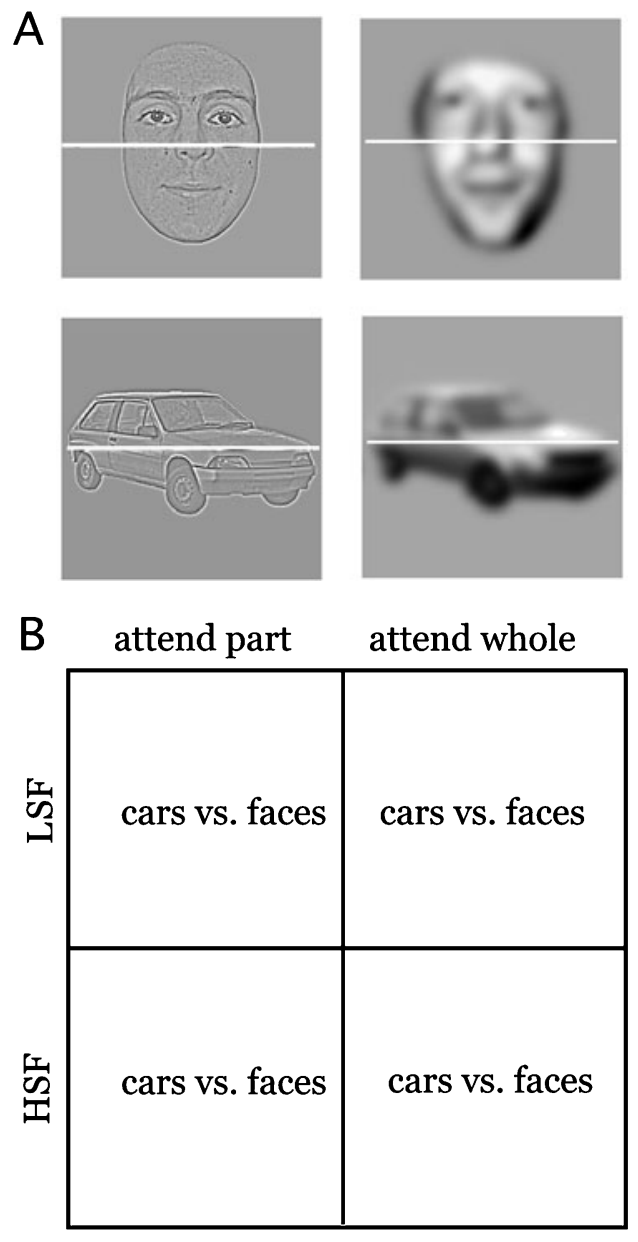

Figure 2. Examples of the stimuli and the experimental design for Experiment 1 (Experiments 2 and 3 used similar images). (A) For Experiments 1 and 2, images of faces and cars were filtered in Matlab, so as to contain only spatial frequencies of $8 \mathrm{cy}-$ cles per image or less (approximately $1.1 \mathrm{cpd}$, LSF stimuli) or 32 cycles per image or more (approximately $4.2 \mathrm{cpd}$, HSF stimuli). In Experiment 3, images of faces in front view and cars (in profile or three quarters view) were shown on a textured background containing a wide range of spatial frequencies and were filtered in the same way (except that the HSF cutoff was 50 cycles per image, or approximately $6.0 \mathrm{cpd}$ ). (B) The experimental runs for each experiment were organized in a three-way factorial design, in all cases showing separate blocks $(12 \mathrm{sec})$ of cars and faces in low spatial frequency (LSF) or high spatial frequency (HSF) format. The third factor differed for each experiment: In Experiment 1 , the subjects were cued to perform one-back identity repetition judgments on either the whole image or only the bottom half of composites; in Experiment 2, the stimuli were presented in one of eight locations, and the subjects were cued prior to each block to perform one-back identity repetition judgments or oneback location repetition judgments; in Experiment 3, the subjects performed one-back identity repetition judgments on either upright or inverted stimuli.

(Vuilleumier, 2000; Winston et al., 2003) or were localized only by contrasting faces with noise stimuli, rather than with object controls (Eger et al., 2004). Here, we defined the FFA functionally in each observer, using standard methods (Gauthier, Skudlarski, et al., 2000; Grill-Spector, Knouf, \& Kanwisher, 2004; Kanwisher et al., 1997) to address more specifically the role of spatial scale in this area. As was expected, standard analytical methods focusing on the mean activity in the FFA revealed similar effects with LSF- and HSF-filtered stimuli. However, a new method focusing on correlations between individual differences across different conditions suggested that the computations taking place in the FFA might not be invariant to such spatial scale information. The results suggest that the FFA receives inputs from separate LSF and HSF channels. The response of each channel seems to be influenced by expertise with objects, but surprisingly, the degree to which each channel predicts behavioral expertise appears to be independent.

\section{METHOD}

\section{Subjects and fMRI Procedures}

All the subjects were healthy right-handed individuals with no history of neurological or psychiatric problems. All the subjects gave written informed consent and were scanned using procedures approved by the Human Investigations Committee at the Yale School of Medicine or the Institutional Review Board at Vanderbilt University.

Experiment 1. Seven adult volunteers (1 female) participated in Experiment 1 in return for monetary compensation. Scanning was performed on a 3-Tesla whole-body GE MRI scanner at the Vanderbilt Medical Center in Nashville. Eighteen contiguous 7-mmthick $(3.75 \times 3.75 \mathrm{~mm}$ in-plane $) \mathrm{T} 1$-weighted anatomical axial images were acquired, parallel to the $\mathrm{AC}-\mathrm{PC}$ line. T2*-weighted images were acquired in the same orientation, using a gradient echo pulse sequence $\left(\mathrm{TR}=2 \mathrm{sec}, \mathrm{TE}=25 \mathrm{msec}\right.$, flip angle $\left.=70^{\circ}\right)$ for blood oxygenation level (BOLD) imaging. The field of view was $24 \times 24 \times 12.6 \mathrm{~cm}$, with an in-plane resolution of $64 \times 64$ pixels, resulting in a voxel size of $3.75 \times 3.75 \times 7.0 \mathrm{~mm}$. Each functional scan lasted $312 \mathrm{sec}$. The first two functional images in each run were discarded, to take into account the equilibrium of longitudinal magnetization.

Experiment 2. Fifteen adult volunteers ( 9 females) participated in Experiment 2 in return for monetary compensation. Scanning was conducted on a 1.5-Tesla whole-body GE MRI scanner at the Vanderbilt Medical Center, using procedures similar to those in Experiment 1 except that 10 axial slices covering the occipital and temporal lobes were obtained and a different pulse sequence was used for T2*-weighted imaging $(\mathrm{TR}=2 \mathrm{sec}$, TE $=60 \mathrm{msec}$, flip angle $\left.=60^{\circ}\right)$. Voxel size was $3.125 \times 3.125 \times 7.0 \mathrm{~mm}$.

Experiment 3. Ten adult volunteers ( 5 females) participated in Experiment 3 in return for monetary compensation. Scanning was conducted on the 1.5-T GE scanner at the Yale School of Medicine, with the same parameters as those in Experiment 2, except that six slices were collected parallel to the line defined by the temporal and occipital poles and covered the occipital and temporal lobes. Each scan lasted $336 \mathrm{sec}$.

\section{Behavioral Procedures}

Experiment 1. Car expertise was measured behaviorally outside of the scanner, using matching judgments for pairs of cars and pairs of birds (Gauthier, Curran, Curby, \& Collins, 2003; Gauthier, Skudlarski, et al., 2000). Briefly, the subjects matched sequentially presented images of different exemplars of cars and birds on the basis of their model or species, across slight changes in viewpoint, color, and illumination (224 trials each). The first image was presented for $1 \mathrm{sec}$ followed by a mask for $500 \mathrm{msec}$, and a second image remained on the screen until a response was made or $5 \mathrm{sec}$ had passed. 
Bird matching provided a baseline for individual differences in object-matching skill (none of the subjects evidenced any special skill with birds).

In an fMRI session, a functional localizer for the FFA was performed using standard procedures (Gauthier, Skudlarski, et al., 2000; Kanwisher et al., 1997). Each subject performed three localizer runs, except for 1 subject, who performed only two localizer runs, to functionally localize the FFA. The runs were constructed using a block design in which grayscale broadpass images of faces or objects were presented centrally for $500 \mathrm{msec}$, with a $500-\mathrm{msec}$ interstimulus interval (ISI), and the subjects performed a one-back identity repetition task. Eight face and eight object blocks (12 sec each) were used, presented alternating with 6-sec fixation blocks (12-sec fixation at the start and end of each scan).

In the same fMRI session, the subjects also performed three experimental runs, using a factorial design with category (car vs. face), spatial frequency content (LSF vs. HSF), and task (whole vs. part judgment) as completely crossed factors. The conditions were presented in blocks of $12 \mathrm{sec}$, with rest blocks $(6 \mathrm{sec})$ preceding each experimental block (12-sec fixation at the start and end of each scan). The order of conditions was counterbalanced between scans, with all eight task conditions (e.g., LSF face whole) performed once before being repeated. In the whole judgment condition, the subjects were required to perform a one-back judgment on the whole image (i.e., the top and bottom of the composite image), whereas in the part-task condition, the subjects were required to perform a same/different judgment on just the bottom of the composite (ignoring the top). The words "whole" or "below" (for below the line) were presented as instructions for the following block during the rest period. For these experimental runs, the stimuli were as follows: Top and bottom halves of 16 faces (different from those used in the localizer) without hair and 16 cars shown in profile on a $50 \%$ gray background were paired to create 32 car composites and 32 face composites. Images were filtered in Matlab to conserve only spatial frequencies of 8 cpi or less (LSF stimuli, approximately 1.1 cycle per degree) and 32 cpi (approximately 4.2 cycles per degree) or more (HSF stimuli). For filtering, a Butterworth filter of order two was used to avoid problems with Gibbs ondulations for hard-limited filters. All the images were clearly recognizable as faces or cars, and each subtended a visual angle of approximately $7.5^{\circ} \times 7.5^{\circ}$ of visual angle. Very low and very high filter cutoffs were chosen to ensure that separate channels would be recruited early in the visual system and would correspond to values that would be expected to yield spatial frequency preferences in face cells according to Rolls et al. (1985). Contrast was not equated across LSF and HSF images, so that the images in each format would contain the same energy that they would contribute to the original image. Each stimulus was presented centrally for $800 \mathrm{msec}$, with a 200 -msec ISI.

Experiment 2. Car expertise was measured behaviorally outside of the scanner, as in Experiment 1, in only 6 of the 15 subjects, 6 months after the fMRI session. In an fMRI session, each subject performed two localizer runs, as in Experiment 1. In addition, the subjects also performed four experimental runs, using a factorial design with category (car vs. face), spatial frequency content (LSF vs. HSF), and task (identity vs. location) as completely crossed factors. The conditions were presented in blocks of $12 \mathrm{sec}$, with fixation blocks $(6 \mathrm{sec})$ preceding each experimental block (12-sec fixation at the start and end of each scan). The order of the conditions was counterbalanced between scans, with all eight task conditions (e.g., LSF face identity) performed once before being repeated. The stimuli for the experimental scans were 36 images of whole cars in profile and 36 images of whole faces without hair on a $50 \%$ gray background. The images were filtered in Matlab, as in Experiment 1 . Each image was presented for $800 \mathrm{msec}$, with a $200-\mathrm{msec}$ ISI, in one of eight possible locations on the screen. The instructions ("LOC" or "OBJ") were presented during the rest period prior to each block. Depending on the instructions only, the subjects were instructed to perform one-back identity or location repetition judgments while ignoring the other dimension. Four experimental scans were performed per subject, with two epochs of $12 \mathrm{sec}$ per scan for each of the eight conditions, plus interspersed rest baselines of $6 \mathrm{sec}$.

Experiment 3. In an fMRI session, each subject performed two localizer runs similar to those in Experiment 1, except that they included scene blocks, as well as faces and objects. In addition, the subjects also performed five experimental runs, using a factorial design with category (car vs. face), spatial frequency content (LSF vs. HSF), and orientation (upright vs. inverted) as completely crossed factors. The order of conditions was counterbalanced between scans. Each scan used an fTfTfTf design, where f stands for a 12sec fixation block and T stands for eight consecutive blocks showing the eight conditions (e.g., LSF faces upright) in a different order each time and counterbalanced across runs. The stimuli for the experimental runs were 36 faces without hair and 36 cars in profile or three quarters view, shown on a textured background containing both LSFs and HSFs. These images were then filtered in Matlab, as in Experiment 1, except that a higher cutoff was used for HSF images (50 cpi, or approximately 6 cycles per degree). Images were presented centrally for $800 \mathrm{msec}$, with a 200 -msec ISI. The subjects performed one-back identity repetition judgments.

\section{Data Analysis}

The first image of each block was discarded from further analysis, and the images were shifted $4 \mathrm{sec}$ relative to stimulus presentation, to account for hemodynamic delay. Functional images showing excessive motion (more than 0.5 pixel over the entire run) were removed from the analysis. The SPM96 algorithm was used to correct for motion between successive images in each run. Statistical parametric maps of BOLD activation of the localizer runs for each subject were created, using a $t$ test comparing the mean activity for face and object conditions, with an additional parameter being used to correct for a linear drift in the signal within each run (Skudlarski, Constable, \& Gore, 1999). These maps were superimposed on anatomical scans. Because of the known variability in the location of the FFA, an individual functional definition of this region was used. An earlier study showed that this method led to stronger face selectivity than a definition based on a group-averaged FFA did (Gauthier, Tarr, Moylan, Anderson, \& Gore, 2000). The FFA was defined functionally for each subject as a contiguous cluster of voxels within the fusiform gyrus (within a search volume extending from the posterior commissure [PC] to $4 \mathrm{~cm}$ posterior to the PC showing stronger activation for faces than for objects, at an arbitrary threshold of $t=1.5$ ). The FFA could be defined in all the subjects in the right hemisphere, but not in the left hemisphere (Experiment 1, 6/7; Experiment 2, 11/15; Experiment 3, 8/10). To maximize power, we will report only the results in the right hemisphere; our claims should therefore be limited to this area. ${ }^{1}$ The percentage of signal change for each condition was calculated for each subject from the rest baseline present in each run, in these FFA regions of interest (ROIs). One-tailed $t$ tests were used to test for a positive correlation of ROI activity with car expertise, because this was a prediction based on Gauthier, Skudlarski, et al. (2000), and two-tailed tests were used for all other comparisons.

\section{RESULTS}

\section{Behavioral Results}

Performance was modulated by the task, in accord with prior work. In Experiment 1, the subjects performed worse when they had to ignore part of the stimuli, revealing holistic processing of both faces and cars (Gau- 
thier et al., 2003), and in Experiment 3, the subjects performed better with upright than with inverted images (Yin, 1969). In addition, the spatial frequency content led to significant differences in behavioral performance in all three experiments (see Table 1). Such differences are consistent with the finding that spatial frequency use depends on the task (Gosselin \& Schyns, 2001; Morrison \& Schyns, 2001; Schyns \& Oliva, 1999). In Experiment 1, there was a significant effect of task on accuracy, with better performance for whole than for bottom judgments $[F(1,6)=33.15, p<.005]$. This could have occurred for one of two reasons: Either the subjects successfully selectively attended to bottom halves and had less information to base their judgments on, or they could not completely selectively attend to the bottom halves (Farah, Wilson, Drain, \& Tanaka, 1998), and interference resulted from the to-be-ignored information present in the top half, which was incongruent with the correct response for the bottom half on $50 \%$ of the trials. Responses were faster for cars than for faces $[F(1,6)=$ $8.80, p<.05]$, as well as for LSF than for HSF images $[F(1,6)=85.88, p<.0001]$. In Experiment 2, accuracy was better for LSF than for HSF images $[F(1,14)=$ $64.14, p<.0001]$. There was also a significant interaction between task and spatial frequency content $[F(1,14)=$ $17.70, p<.001]$, as well as an unexpected three-way interaction of these factors with category $[F(1,14)=$ $12.30, p<.005]$, which was produced by significantly better performance for LSF than for HSF judgments in all the conditions, except for faces in the location task. LSF stimuli may afford an advantage for location judgments, because the HSF information is less useful for estimating the center of mass of each object and this may be less important for faces, for which the silhouette was more regular than that of cars. Responses were faster in the location than in the identity task $[F(1,14)=7.96$, $p<.05]$, as well as for LSF than for HSF images, $[F(1,14)=38.39, p<.0001]$. In Experiment 3, accuracy was better with HSF than with LSF images ${ }^{2}[F(1,13)=$ $35.00, p<.0001]$ and was better for upright than for in- verted images $[F(1,13)=12.31, p<.005]$. There were no significant differences in response times.

\section{FFA Responses Averaged Across Subjects}

The right FFA (hereafter, FFA) - localized using broadband stimuli - was selective for faces (relative to cars) for both HSF and LSF stimuli. Despite the fact that spatial frequency content influenced behavior in all the experiments, it had no influence on mean activity in the FFA. For each experiment, the mean percentage of signal change in the FFA across subjects, for each condition relative to fixation, is plotted in Figure 3. In general, there were large significant effects of category and smaller, but still significant, effects of task (or orientation), regardless of spatial scale. In Experiment 1, there was more activity for faces than for cars $[F(1,6)=6.7$, $p<.005]$. However, it can be appreciated in Figure 3 that the difference in activity between faces and cars was much less in Experiment 1 than in the other two experiments. This likely was caused by the fact that several subjects in Experiment 1 had very strong car expertise (in contrast, the subjects in Experiments 2 and 3 were not recruited for any special aptitude with cars). No other main effect or interaction was significant in Experiment 1. In Experiment 2, there was more activity for faces than for cars $[F(1,15)=27.93, p<.0001]$, as well as more activity during identity than during location judgments $[F(1,15)=10.33, p<.01]$. No other main effect or interaction was significant. In Experiment 3, we again found more activity for faces than for cars $[F(1,9)=$ $18.25, p<.002$ ], as well as more activity for upright than for inverted images $[F(1,9)=6.32, p<.05]$. No other main effect or interaction was significant.

In sum, we found no significant effect of spatial frequency content on the mean response in the FFA in any experiment.

These results are in general agreement with the idea that selectivity in the FFA is relatively insensitive to spatial scale. To investigate this question further, we focus on an interesting property of FFA activity: its correlation

Table 1

Mean Accuracy and Reaction Time for Correct Responses (With Standard Errors of the Means) During Experiments 1-3

\begin{tabular}{|c|c|c|c|c|c|c|c|c|c|c|c|c|}
\hline \multirow[b]{3}{*}{ Measure } & \multicolumn{4}{|c|}{ Experiment 1} & \multicolumn{4}{|c|}{ Experiment 2} & \multicolumn{4}{|c|}{ Experiment 3} \\
\hline & \multicolumn{2}{|c|}{ Whole } & \multicolumn{2}{|c|}{ Bottom } & \multicolumn{2}{|c|}{ Identity } & \multicolumn{2}{|c|}{ Location } & \multicolumn{2}{|c|}{ Upright } & \multicolumn{2}{|c|}{ Inverted } \\
\hline & $M$ & $\overline{S E M}$ & $M$ & $S E M$ & $M$ & $\overline{S E M}$ & $M$ & $\overline{S E M}$ & $M$ & $S E M$ & $M$ & SEM \\
\hline \multicolumn{13}{|l|}{ Accuracy } \\
\hline Car HSF & .82 & .04 & .80 & .03 & .85 & .02 & .81 & .02 & .87 & .02 & .83 & .02 \\
\hline Car LSF & .88 & .03 & .79 & .02 & .87 & .01 & .86 & .01 & .82 & .03 & .76 & .03 \\
\hline Face HSF & .86 & .02 & .75 & .03 & .82 & .01 & .87 & .01 & .85 & .03 & .80 & .02 \\
\hline Face LSF & .87 & .03 & .79 & .02 & .87 & .01 & .86 & .02 & .80 & .03 & .77 & .02 \\
\hline \multicolumn{13}{|c|}{ Reaction Time (msec) } \\
\hline Car HSF & 545 & 23 & 522 & 15 & 551 & 12 & 520 & 12 & 520 & 14 & 530 & 13 \\
\hline Car LSF & 491 & 11 & 495 & 13 & 518 & 14 & 506 & 15 & 537 & 15 & 560 & 17 \\
\hline Face HSF & 546 & 10 & 552 & 12 & 548 & 13 & 530 & 16 & 534 & 13 & 545 & 18 \\
\hline Face LSF & 503 & 13 & 515 & 14 & 511 & 14 & 501 & 19 & 539 & 15 & 555 & 13 \\
\hline
\end{tabular}

Note-HSF, high spatial frequency; LSF, low spatial frequency. 

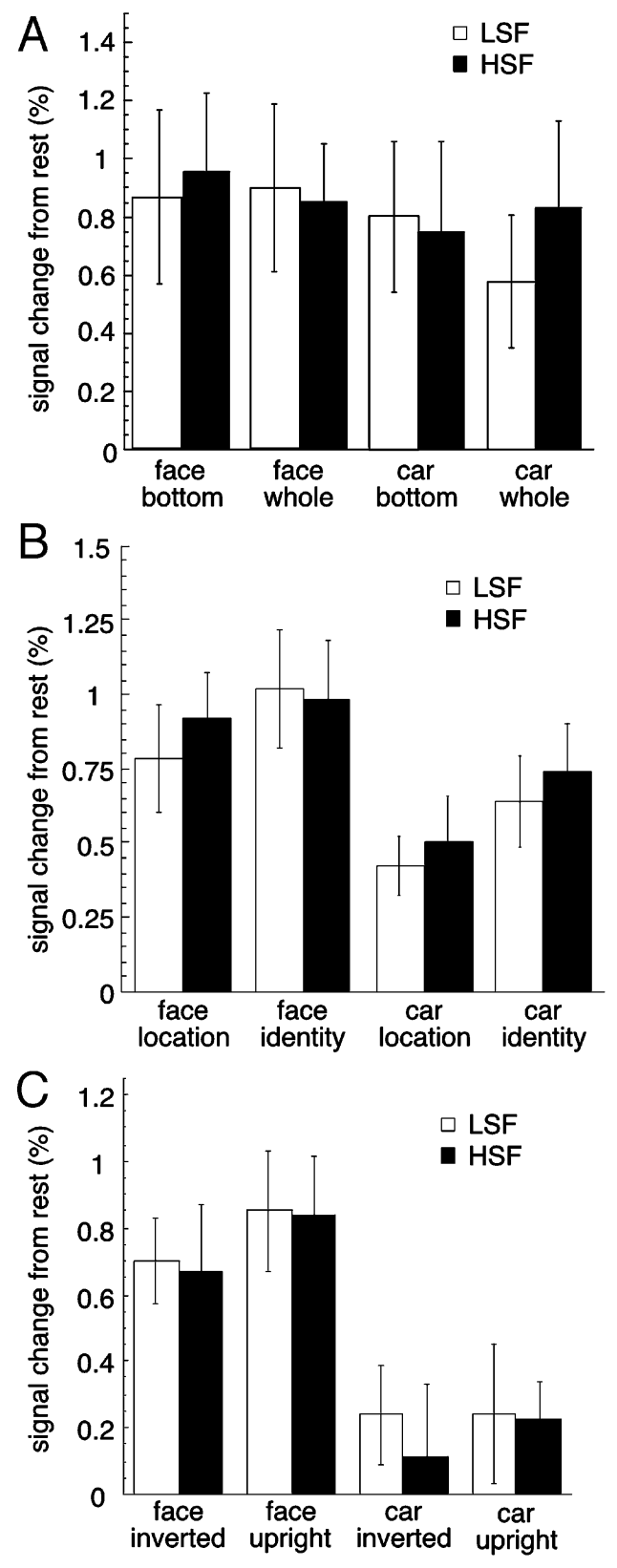

Figure 3. Mean percentage of signal change in each condition, relative to a fixation baseline in the right fusiform face area region of interest, for Experiment 1 (A), Experiment 2 (B), and Experiment $3(C)$. Error bars show the standard errors of the means. LSF, low spatial frequency; HSF, high spatial frequency.

with individual differences in expertise with an object category (Gauthier, Skudlarski, et al., 2000). A simple prediction is that if processing in the FFA is invariant to spatial scale, expertise should predict FFA responses for both HSF and LSF images. We first will report these correlations with behavioral expertise; then we will test a second prediction of a scale invariant model: that individual differences in FFA selectivity should be correlated for HSF and LSF stimuli.

\section{Correlations of fMRI Data With Behavior}

In previous studies, FFA activity correlated with a subject's level of expertise with a category-for example, with car or bird experts (Gauthier, Skudlarski, et al., 2000; Xu, in press) or with subjects trained to expertise with novel objects (Gauthier \& Tarr, 2002). In Experiment 1 , we recruited subjects with a range of expertise (from none to extensive) with cars and measured FFA responses to cars and faces, when shown LSF and HSF versions. Sensitivity for car judgments varied between $d^{\prime}$ s of 0.65 and 3.76 (corresponding to $62.5 \%-98.21 \%$ accuracy), and sensitivity for bird judgments varied between a $d^{\prime}$ s of 0.35 and 1.32 (corresponding to $57.1 \%$ $75 \%$ accuracy). The $\Delta d^{\prime}$ for cars versus birds was used as a measure of car expertise, normalized for individual differences in subordinate-level judgments in a novice category. Car expertise was correlated with the response to cars minus that to faces in the FFA for HSF images $[r=.70 ; F(1,6)=4.66, p<.05]$, but not for LSF images $[r=.47 ; F(1,6)=1.39$, n.s. $]$. However, in a multiple regression in which the regression coefficients represent the independent contributions of each spatial scale to car expertise, both HSF and LSF were significant predictors (both $p \mathrm{~s}<.05$ ), and the two-predictor model was able to account for $85.2 \%$ of the variance in expertise $[F(2,6)=$ $11.5, p<.05$; see Figures 4A and 4B]. Note that car activity approached or even exceeded face activity in the subjects with the highest levels of car expertise, suggesting that the response to objects of expertise can surpass that for faces in the best object experts. In Experiment 2, the subjects were not recruited with regard to experience with cars, but car expertise scores were gathered later for 6 of the subjects, approximately 6 months after the fMRI sessions. Despite the limited range of behavioral expertise in this small sample, a direct relationship between car expertise and FFA activity for cars minus faces was obtained $[r=.96 ; F(1,5)=42.7, p<$ .005 ; Figure 4D]. Surprisingly, neither a large range of car expertise nor an awareness that the experiment concerns car expertise is necessary for a robust relationship. In this case, the correlation of expertise with LSF fMRI results (for cars minus faces) was significant $[r=.90$; $F(1,5)=16.3, p<.02]$, but not that with the same comparison for HSF fMRI results $[r=.46 ; F(1,5)=1.04$, n.s.]. However, again, both LSF and HSF contrasts were significant predictors of car expertise (both $p \mathrm{~s}<.05$ ) in a multiple regression, and the two-predictor model accounted for $93.6 \%$ of the variance. Car expertise scores were not obtained in Experiment 3.

Is this relationship between fMRI activity and car expertise specific to the FFA? Using the data from Experiment 1 (in which we had the largest range of car expertise), we performed the same analysis in two objectselective regions of the parahippocampal gyrus (PHG) and lateral occipital cortex (LOC; regions defined individually in the localizer runs as responding more to objects than faces; they also responded more to cars than to faces in experimental runs). In these areas, there was no 

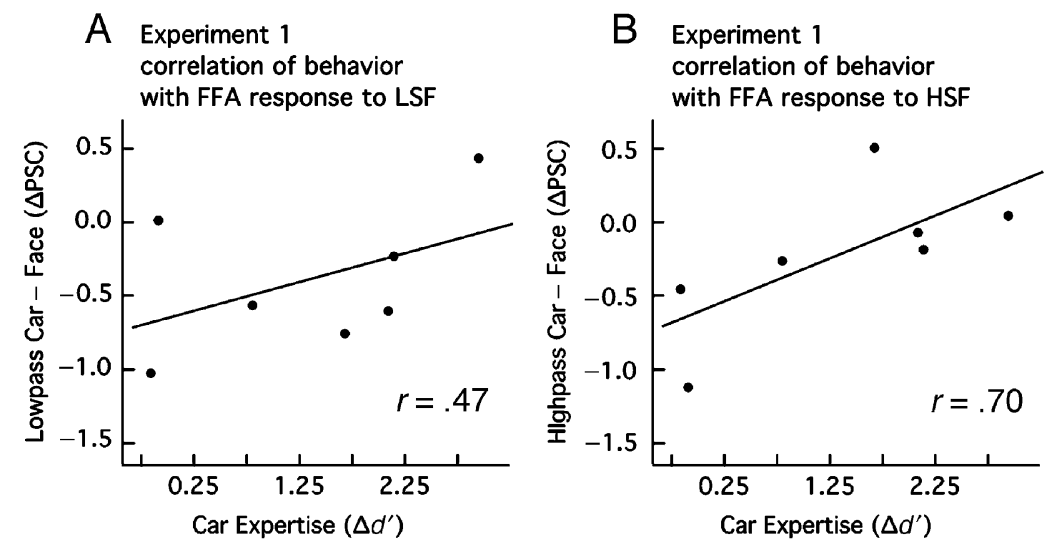

C Experiment 1 correlation of behavior with FFA response to LSF+HSF

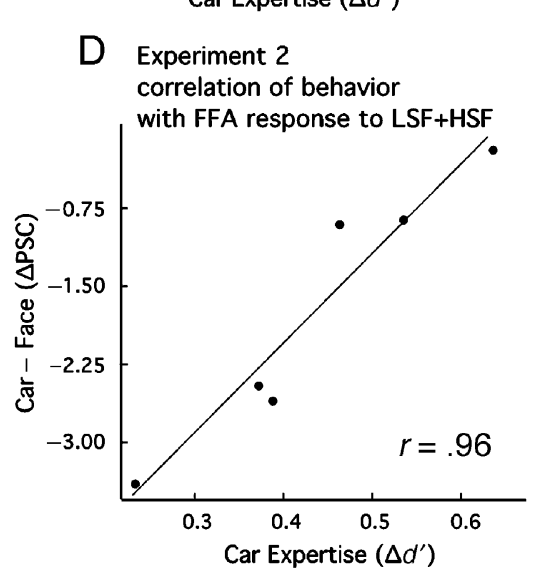

Figure 4. Correlations between a behavioral measure of car expertise and right fusiform face area (FFA) activity. A behavioral measure of car expertise is related to activity in the right FFA for cars minus faces with (A) low spatial frequency (LSF) images and (B) high spatial frequency (HSF) images in Experiment 1. Although only the correlation with FFA activity for HSF images reached significance, a multiple regression showed activity for cars minus faces, for both HSF and LSF, to be reliable predictors of behavioral car expertise (see the text). (C) Correlation between behavioral expertise and FFA activity for cars minus faces for both LSF and HSF images, averaged together in Experiment 1. (D) Correlation between FFA activity for cars minus faces for both LSF and HSF images, averaged together in Experiment 2. In Experiment 2, the correlation with expertise was higher for LSF images than for HSF images, but both were significant predictors of car expertise in a multiple regression (see the text).

clear relationship between car expertise and activity to cars versus faces, either for each spatial frequency condition separately or when averaged (average results: right PHG, $r=.35, p=.75$; right LOC, $r=-.20, p=.67$ ). Thus, car expertise was found to be related to fMRI activity in face-selective, but not in car-selective, areas.

\section{Correlations of Individual Differences in fMRI Data Across Different Conditions}

To summarize, both LSF and HSF images evoke similar face-selective responses in the FFA, and car expertise is largely predicted by the combined response to LSF and HSF stimuli. One possible interpretation of these results is that cells in the FFA are invariant to spatial scale. However, if this is the case, individual differences in brain activation for LSF images will be expected to pre- dict individual differences for HSF images. In other words, if one individual shows much more FFA activity for cars than for faces with LSF images, this person should also show much more FFA activity for cars than for faces with HSF images.

However, across all three experiments, we found that the selectivity for cars versus faces in LSF and HSF images was not significantly correlated across subjects (Figures 5A, 5C, and 5E). The lack of correlation of FFA responses between the two spatial frequency conditions can be contrasted with the clear relationship of FFA responses between the two different tasks or conditions in each experiment (Figures 5B, 5D, and 5F). In Experiment 1 , the subjects performed one-back identity judgments on wholes versus parts in different epochs. In Experiment 2 , the subjects attended to the identity or the 
A Experiment 1 correlation between SF conditions

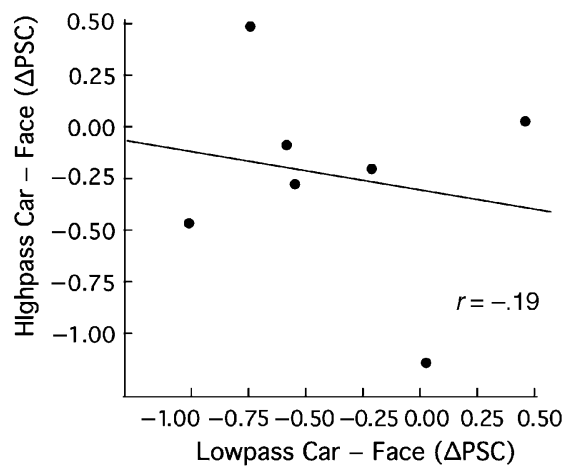

C Experiment 2

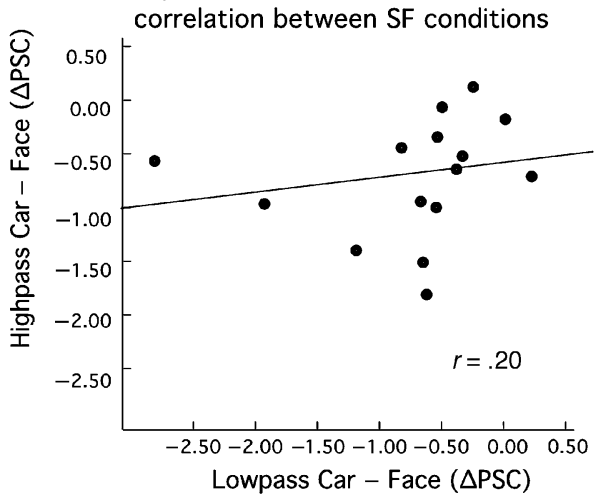

E Experiment 3

correlation between SF conditions

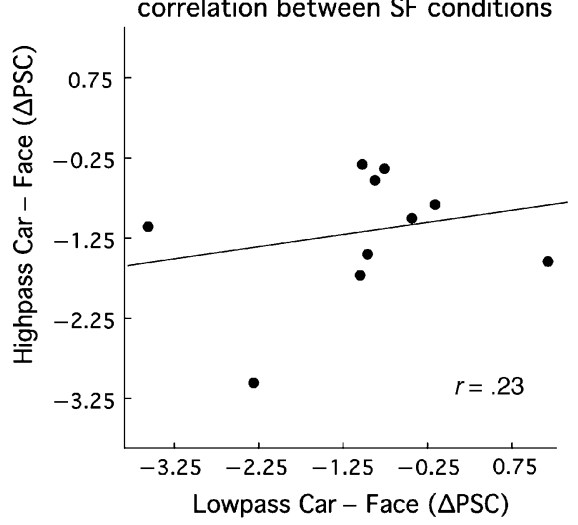

B Experiment 1 correlation between task conditions

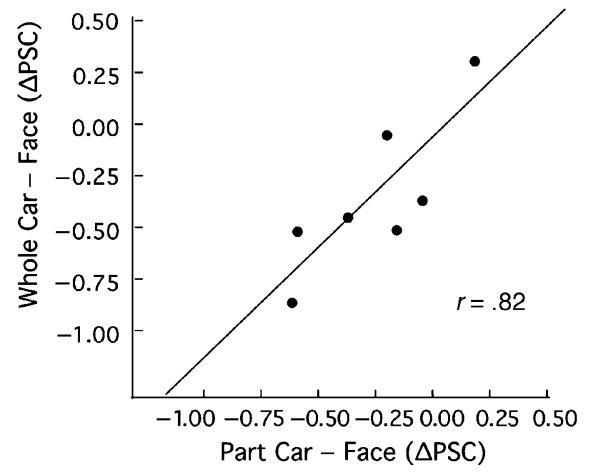

D Experiment 2

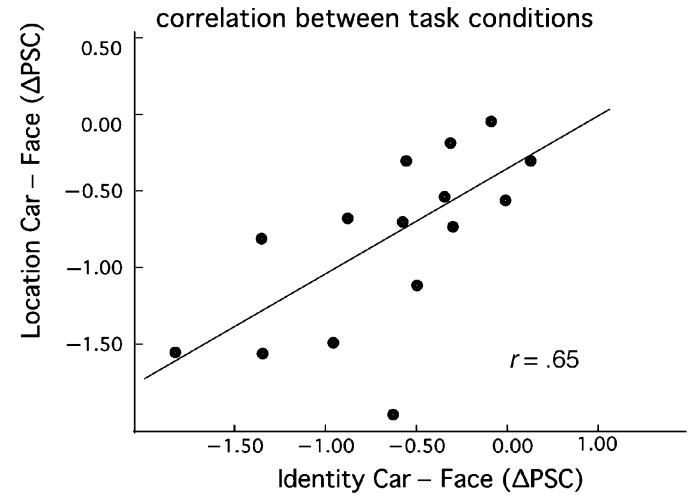

F Experiment 3

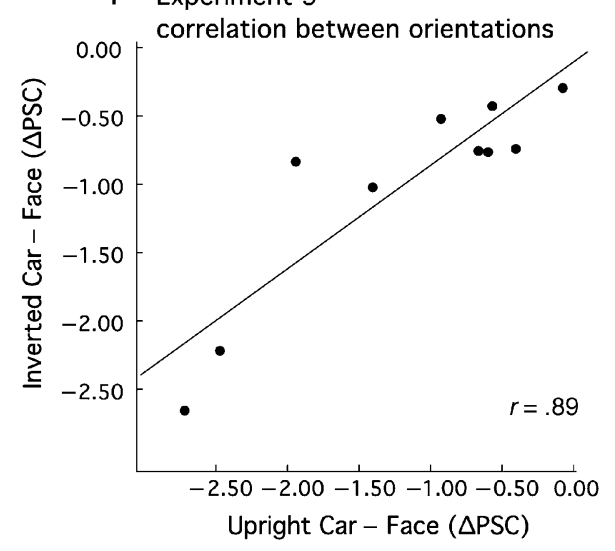

Figure 5. Correlations of individual differences of fusiform face area (FFA) activity between different spatial scales and different tasks. In Experiment 1, (A) FFA activity for cars minus faces in low-pass (low spatial frequency, LSF) images is poorly correlated with right FFA activity for cars minus faces with highpass (high spatial frequency, HSF) images $[r=-.19 ; F(1,6)=0.18$, n.s. $]$, but $(B)$ a high correlation is obtained in the same data set for the orthogonal analysis on the correlation of individual differences in car minus face activity between the two tasks [attend whole vs. attend part: $r=.82 ; F(1,6)=10.5, p=.02]$. In Experiment 2, (C) FFA activity for cars minus faces shown in LSF is poorly correlated with right FFA activity for cars minus faces shown in HSF $[r=.20 ; F(1,14)=0.54$, n.s. $]$, but (D) a high correlation is obtained in the same data set for the orthogonal analysis on the correlation of individual differences in car minus face activity between the two tasks [attend location vs. identity: $r=.65 ; F(1,14)=9.58, p=.01]$. In Experiment 3, (E) FFA activity for cars minus faces shown in LSF is poorly correlated with right FFA activity for cars minus faces shown in HSF $[r=.23 ; F(1,9)=0.46$, n.s. $]$, but $(F)$ a high correlation is obtained in the same data set for the orthogonal analysis on the correlation of individual differences in car minus face activity between the two tasks [attend location vs. identity: $r=.89 ; F(1,9)=30.5, p=.001$ ]. Note that the results are collapsed over the two tasks in each experiment in panels $\mathrm{A}, \mathrm{C}$, and $\mathrm{E}$ and are collapsed over spatial frequency in panels $B, D$, and $F$. 
location of the images in different epochs. In Experiment 3 , the subjects made judgments about the identity of images presented either upright or upside down in different epochs. In all three experiments, the FFA response to cars versus faces in one task condition could be predicted from the response in the other task condition (e.g., attention to parts or to wholes). Therefore, the lack of correlation for individual FFA responses in different spatial scales is not a methodological artifact: Using the same data analyzed in an orthogonal fashion, we find that individual FFA responses are highly replicable across measurements when images contain the same spatial frequencies.

In these analyses, correlations of car versus face activity in the FFA were calculated between two conditions, collapsing across another manipulation (e.g., in Experiment 1, in the correlation between the LSF and the HSF conditions, each value was an average of the percentage of signal change for the whole and the bottom conditions). There is a chance that such aggregate correlations could be misleading, because of Simpson's paradox (a possible reversal of the direction of a comparison or an association when data from several groups are combined to form a single group; Moore \& McCabe, 1999). To guard against this paradox and to provide a better estimate of the effects obtained in the three experiments, the nonaggregate correlations (e.g., the correlation between car minus face activity in LSF format and car minus face activity in HSF format, only in the attendwhole condition) from all three experiments were combined using standard meta-analytical methods (Hunter, 1982). First, the correlation coefficients for each study were weighted by sample size, and a Fisher's transformation was applied, before averaging values from the three experiments and computing a 95\% confidence interval. The average correlation between the LSF and the HSF conditions was $r=.05$, with a $95 \%$ confidence interval of $r=-.23$ to $r=.32$, and therefore, was not significantly different from 0 . In contrast, when analyses were limited to one of the spatial frequency conditions (correlations between different tasks, but within the same spatial frequency condition), the average correlation was significantly higher than $0(r=.68)$, with a confidence interval ranging from $r=.37$ to $r=.74$. Crucially, the two confidence intervals do not overlap, revealing that the correlation across task/orientation is higher than that across spatial scales. These results suggest relatively independent processing at different spatial scales within the FFA.

\section{DISCUSSION}

\section{Processing Images at Different Spatial Scales in the FFA}

The most surprising and novel result in this study is evidence that processing of LSF and HSF images in the FFA are relatively independent (although complete independence is unlikely, given that there is a small but significant degree of mutual inhibition between channels in the striate cortex; De Valois \& Tootell, 1983). We draw this conclusion primarily on the basis of the absence of a correlation between individual differences in selectivity in the FFA for LSF and HSF images. By itself, the lack of a correlation across different spatial scales is a null result and, as such, may be difficult to interpret. However, we also obtained the following evidence in support of this finding. First, the absence of a correlation across channels was obtained in three separate experiments, which differed in many aspects of the method and pattern of behavioral performance, so the results are unlikely to have been attached to a spurious manipulation or correlation with task difficulty. Second, the absence of replicable individual differences across spatial scales was contrasted with three other manipulations that led to robust correlations, within the same data sets, so the results are unlikely to have been due to a lack of power or to our statistical procedures. Third, a meta-analysis confirmed that correlations across control conditions were higher than those across changes in spatial scale, casting the results in terms of a positive finding, rather than a null effect.

As is discussed in Figure 1, although the absence of a correlation between HSF and LSF is difficult to interpret statistically, as are all null effects, this pattern is relatively easier to interpret than a significant correlation would be. Indeed, it is not clear that the positive correlations we observed across tasks necessarily indicate that the same cell population mediates performance in both tasks. Consider the example of the positive correlation obtained in Experiment 3 between individual differences in the processing of upright and inverted stimuli. Although fMRI studies indicate that the FFA responds to both upright and inverted faces (Haxby et al., 1999; Kanwisher et al., 1998), individual face cells are orientation sensitive (Hasselmo, Rolls, Baylis, \& Nalwa, 1989; Perrett et al., 1991; Perrett et al., 1985). Thus, in this case, the correlation for individual differences for upright and inverted stimuli is unlikely to indicate that the same cell population is involved in the processing of both orientations. A possible explanation, pointed out by a reviewer, is that behavioral car expertise is the source of the betweensubjects variability that leads to correlations in both tasks. We cannot test this idea for Experiment 3, because we lack a measure of car expertise. However, it is worth noting that, in support of this hypothesis, the correlation between the two tasks for those subjects for which we have a measure of car expertise was considerably reduced in Experiment 1 and switched from positive to negative in Experiment 2, when car expertise was partialed out (from $r=.82$ to $r=.32$ in Experiment 1; from $r=.64$ to $r=-.53$ in Experiment 2). This account could be explored in future studies with a larger sample of subjects tested for car expertise. One possible reason for the correlation between tasks, and not between spatial frequency conditions, is that the distributions of cells responding to different spatial frequency bands are established earlier in development than the distribution for 
orientation selectivity (and may differ across individuals, so that some individuals have more HSF than the LSF cells and vice versa). Orientation selectivity is thought to be based on frequency of encounter (Perrett, Oram, \& Ashbridge, 1998), so the pattern of results we obtain could be due to similar proportions of cells being recruited for different orientations among the HSF and the LSF populations. The same explanation could account for the fact that both HSF and LSF populations correlate with car expertise, another aspect of selectivity likely to develop after spatial frequency tuning. These relative distributions of HSF and LSF cells across individuals could potentially impact what information is best extracted during the acquisition of expertise, although this is highly speculative.

An important caveat is that in our experiment, we did not use the middle frequencies (8-16 cpi) that are thought to best support face perception (Gold, Bennett, \& Sekuler, 1999; Liu et al., 2000). However, our findings nevertheless appear to be quite relevant to behavioral performance, given that BOLD responses for these highly filtered images accounted for the major part of the variance in individual differences for car recognition.

A number of psychophysical studies have revealed the flexibility of the systems responsible for object and face perception with respect to the use of different spatial scales. For example, observers can bias their use of LSF or HSF information, using attention to meet the demands of different recognition tasks (Gosselin \& Schyns, 2001; Morrison \& Schyns, 2001; Schyns \& Oliva, 1999). Gold et al. (1999) compared letter and face identification with that for an ideal observer and found important differences in subjects' efficiencies with these two classes (e.g., the subjects could identify one-octave filtered letters, but not faces at most spatial scales, even though information was available to the ideal observer for both categories). These differences could not be explained by low-level constraints and, thus, indicate that observers sample faces by using a nonoptimal strategy, one that may reflect the need to support not only identification, but also other tasks, such as expression recognition. It is likely that similar spatial-sampling biases can develop when observers acquire expertise with a category and demonstrate face-like processing of these objects (Gauthier \& Tarr, 2002).

Our findings suggest that the flexibility in using different spatial scales in different situations could take place at least as far in the visual stream as the FFA. This area also seems to be especially well suited to fine tuning resulting from experience with specific tasks, given its already demonstrated role in perceptual expertise (Gauthier, Skudlarski, et al., 2000; Gauthier et al., 1999). In this study, the relationship between car expertise and FFA activity for LSF and HSF images suggests that experience with cars may have tuned the activity of cells selective for distinct spatial scales in the FFA, potentially increasing the ability to discriminate objects across a range of distances and viewing conditions.

\section{Implications for the Expertise Model of FFA Activity}

Our results confirmed prior findings showing that activity in the FFA is related to expertise with nonface objects (Gauthier, Skudlarski, et al., 2000; Gauthier et al., 1999; Xu, in press). We replicated and extended these results by showing that BOLD responses to HSF and LSF stimuli make separate contributions in accounting for car expertise. In addition, in Experiment 2, FFA activity predicted car-matching skills in novices tested behaviorally 6 months after the fMRI session. These subjects were unaware that aptitude at car recognition was of interest when they were scanned. This argues against the idea that the relationship between FFA activity and expertise is mediated by strategic or attentional factors. Although an attentional account of expertise effects in the FFA has been offered (Kanwisher, 2000), recent work has revealed that attention increases the neural selectivity of neural populations in the ventral visual system and, therefore, is unlikely to result in the recruitment of faceselective areas for nonface objects (Murray \& Wojciulik, 2004). Such findings make it difficult to "explain away" expertise effects for nonface objects, using an attentional account.

In addition, we find that it is the activity in faceselective areas (and not the activity in areas such as the PHG or the LOC, which responded more to cars than to faces) that is correlated with car expertise. Although this is difficult to explain within a modular framework, in which the FFA is thought of as a face-specific processor (Kanwisher, 2000), it is a direct prediction of an expertise model (Tarr \& Gauthier, 2000) that emphasizes the type of processing subjects learn to apply to a category, rather than its geometry.

\section{A Method for Investigating the Integration of Information in the Brain}

The new method described here, based on correlations of individual differences in brain activity across different tasks or stimulus conditions, could be extended to other problems in cognitive neuroscience, to investigate the locus of integration for different sources of information. An interesting avenue may be to consider how these correlations vary over large expanses of the cortex: Early cortical stages should lead to small correlations when information is processed relatively independently, but much stronger correlations should be obtained in areas downstream from the point of integration. Exploratory analyses with our data suggest that the correlation between different tasks is higher than the correlation between different spatial frequencies in areas upstream from the FFA in the visual cortex but that the correlations between tasks and between spatial frequencies are more comparable in areas that would be more likely downstream from the FFA. This approach would benefit from a larger sample size and from the ability to register different brains on the basis of functional, as well as anatomical, landmarks. 


\section{REFERENCES}

Blakemore, C., \& Campbell, F. W. (1969). On the existence of neurones in the human visual system selectively sensitive to the orientation and size of retinal images. Journal of Physiology, 203, 237-260.

Bruner, J. S., \& Potter, M. C. (1964). Interference in visual recognition. Science, 144, 424-425.

CAmpbell, F. W., \& Robson, J. R. (1968). Application of Fourier analysis to the visibility of gratings. Journal of Physiology, 197, 551-566.

De Valois, K. K., \& Tootell, R. B. (1983). Spatial-frequency-specific inhibition in cat striate cortex cells. Journal of Physiology, 336, 359-376.

Eger, E., Schyns, P. G., \& Kleinschmidt, A. (2004). Scale invariant adaptation in fusiform face-responsive regions. NeuroImage, 22, 232-242.

Farah, M.-J., Wilson, K.-D., Drain, M., \& TANaKa, J.-N. (1998). What is "special" about face perception? Psychological Review, 105, 482-498.

Gauthier, I., Curran, T., Curby, K. M., \& Collins, D. (2003). Perceptual interference supports a nonmodular account of face processing. Nature Neuroscience, 6, 428-432.

Gauthier, I., Skudlarski, P., Gore, J. C., \& Anderson, A. W. (2000). Expertise for cars and birds recruits brain areas involved in face recognition. Nature Neuroscience, 3, 191-197.

GAUTHIER, I., \& TARR, M. J. (2002). Unraveling mechanisms for expert object recognition: Bridging brain activity and behavior. Journal of Experimental Psychology: Human Perception \& Performance, 28, 431-446.

Gauthier, I., Tarr, M. J., Anderson, A. W., Skudlarski, P., \& Gore, J. C. (1999). Activation of the middle fusiform "face area" increases with expertise in recognizing novel objects. Nature Neuroscience, $\mathbf{2}$, 568-573.

Gauthier, I., Tarr, M. J., Moylan, J., Anderson, A. W., \& Gore, J. C. (2000). Does subordinate-level categorization engage the functionallydefined fusiform face area? Cognitive Neuropsychology, 17, 143-163.

Gilinsky, A. S. (1968). Orientation-specific effects of patterns of adapting light on visual acuity. Journal of the Optical Society of America, 58, 13-18.

Gold, J., Bennett, P. J., \& Sekuler, A. B. (1999). Identification of band-pass filtered letters and faces by human and ideal observers. $\mathrm{Vi}$ sion Research, 39, 3537-3560.

Gosselin, F., \& Schyns, P. G. (2001). Bubbles: A technique to reveal the use of information in recognition tasks. Vision Research, 41, 2261-2271.

Grill-Spector, K., KnOuf, N., \& Kanwisher, N. (2004). The fusiform face area subserves face perception, not generic within-category identification. Nature Neuroscience, 7, 555-562.

Grill-Spector, K., Kushnir, T., Edelman, S., Avidan, G., ItZchaK, Y., \& MalaCH, R. (1999). Differential processing of objects under various viewing conditions in the human lateral occipital complex. $\mathrm{Neu}$ ron, 24, 187-203.

Hasselmo, M. E., Rolls, E. T., Baylis, G. C., \& Nalwa, V. (1989) Object-centered encoding by face-selective neurons in the cortex in the superior temporal sulcus of the monkey. Experimental Brain Research, 75, 417-429.

Hasson, U., Harel, M., Levy, I., \& Malach, R. (2003). Large-scale mirror-symmetry organization of human occipito-temporal object areas. Neuron, 37, 1027-1041.

Haxby, J. V., Ungerleider, L. G., Clark, V. P., Schouten, J. L., Hoffman, E. A., \& Martin, A. (1999). The effect of face inversion on activity in human neural systems for face and object perception. Neuron, 22, 189-199.

Henson, R., Shallice, T., \& Dolan, R. (2000). Neuroimaging evidence for dissociable forms of repetition priming. Science, $\mathbf{2 8 7}$, 1269-1272.

Hess, R. F. (2004). Spatial scale in visual processing. In L. M. Chalupa \& J. S. Werner (Eds.), The visual neurosciences (pp. 1043-1059). Cambridge, MA: MIT Press.

Hunter, J. E. (1982). Meta-analysis: Cumulating research findings across studies. Beverly Hills, CA.: Sage.
Ishai, A., Ungerleider, L. G., Martin, A., Schouten, J. L., \& HAXвY, J. (1999). Distributed representation of objects in the human ventral visual pathway. Proceedings of the National Academy of Sciences, 96, 9379-9384.

KANWISHER, N. (2000). Domain specificity in face perception. Nature Neuroscience, 3, 759-763.

Kanwisher, N., McDermott, J., \& Chun, M. M. (1997). The fusiform face area: A module in human extrastriate cortex specialized for face perception. Journal of Neuroscience, 17, 4302-4311.

Kanwisher, N., Tong, F., \& Nakayama, K. (1998). The effect of face inversion on the human fusiform face area. Cognition, 68, B1-B11.

LegGe, G. E., \& Foley, J. M. (1980). Contrast masking in human vision. Journal of the Optical Society of America, 70, 1458-1471.

Lerner, Y., Hendler, T., Ben-Bashat, D., Harel, M., \& Malach, R. (2001). A hierarchical axis of object processing stages in the human visual cortex. Cerebral Cortex, 11, 287-297.

Levy, I., Hasson, U., Avidan, G., Hendler, T., \& Malach, R. (2001). Center-periphery organization of human object areas. Nature Neuroscience, 4, 533-539.

Liu, C. H., Collin, C. A., Rainville, S. J., \& Chaudhuri, A. (2000). The effects of spatial frequency overlap on face recognition. Journal of Experimental Psychology: Human Perception \& Performance, 26, 956-979.

Malach, R., Reppas, J. B., Benson, R. R., Kwong, K. K., Jiang, H., Kennedy, W. A., Ledden, P. G., Brady, T. J., Rosen, B. R., \& Tootell, R. B. (1995). Object-related activity revealed by functional magnetic resonance imaging in human occipital cortex. Proceedings of the National Academy of Sciences, 92, 8135-8139.

McCarthy, G., Puce, A., Belger, A., \& Allison, T. (1999). Electrophysiological studies of human face perception: II. Response properties of face-specific potentials generated in occipitotemporal cortex. Cerebral Cortex, 5, 431-444.

McCarthy, G., Puce, A., Gore, J. C., \& Allison, T. (1997). Facespecific processing in the human fusiform gyrus. Journal of Cognitive Neuroscience, 9, 605-610.

Moore, D., \& McCABE, G. (1999). Introduction to the practice of statistics. New York: Freeman.

Morrison, D. J., \& SchYns, P. G. (2001). Usage of spatial scales for the categorization of faces, objects, and scenes. Psychonomic Bulletin \& Review, 8, 454-469.

Movshon, J. A., Thompson, I. D., \& Tolhurst, D. J. (1978). Spatial and temporal contrast sensitivity of neurones in areas 17 and 18 of the cat's visual cortex. Journal of Physiology, 283, 101-120.

Murray, S. O., \& Wojciulik, E. (2004). Attention increases neural selectivity in the human lateral occipital complex. Nature Neuroscience, 7, 70-74.

Perrett, D. I., Oram, M. W., \& Ashbridge, E. (1998). Evidence accumulation in cell populations responsive to faces: An account of generalisation of recognition without mental transformations. Cognition, 67, 111-145.

Perrett, D. I., Oram, M. W., Harries, M. H., Bevan, R., Hietanen, J. K., Beson, P. J., \& Thomas, S. (1991). Viewer-centred and objectcentred coding of heads in the macaque's temporal cortex. Experimental Brain Research, 86, 159-173.

Perrett, D. I., Smith, P. A. J., Potter, D. D., Mistlin, A. J., Head, A. S., Milner, A. D., \& Jeeves, M. A. (1985). Visual cells in the temporal cortex sensitive to face view and gaze direction. Proceedings of the Royal Society of London: Series B, 223, 293-317.

Puce, A., Allison, T., Gore, J. C., \& McCarthy, G. (1995). Facesensitive regions in human extrastriate cortex studied by functional MRI. Journal of Neurophysiology, 74, 1192-1199.

Robson, J. G., Tolhurst, D. J., Freeman, R. D., \& Ohzawa, I. (1988). Simple cells in the visual cortex of the cat can be narrowly tuned for spatial frequency. Visual Neuroscience, 1, 415-419.

Rolls, E. T., BAylis, G. C., \& LeOnARD, C. M. (1985). Role of low and high spatial frequencies in the face-selective responses of neurons in the cortex in the superior temporal sulcus in the monkey. Vision Research, 25, 1021-1035.

Sasaki, Y., Hadjikhani, N., Fischl, B., LiU, A. K., Marrett, S., 
Dale, A. M., \& Tootell, R. B. (2001). Local and global attention are mapped retinotopically in human occipital cortex. Proceedings of the National Academy of Sciences, 98, 2077-2082.

Schyns, P. G., Bonnar, L., \& Gosselin, F. (2002). Show me the features! Understanding recognition from the use of visual information. Psychological Science, 13, 402-409.

Schyns, P. G., \& Oliva, A. (1999). Dr. Angry and Mr. Smile: When categorization flexibly modifies the perception of faces in rapid visual presentations. Cognition, 69, 243-265.

Sergent, J., Ohta, S., \& MacDonald, B. (1992). Functional neuroanatomy of face and object processing: A positron emission tomography study. Brain, 115, 15-36.

Skudlarski, P., Constable, R. T., \& Gore, J. C. (1999). ROC analysis of statistical methods used in functional MRI: Individual subjects. NeuroImage, 9, 311-329.

TARr, M. J., \& GAUThIER, I. (2000). FFA: A flexible fusiform area for subordinate-level visual processing automatized by expertise. Nature Neuroscience, 3, 764-769.

Tsao, D. Y., Freiwald, W. A., Knutsen, T. A., Mandeville, J. B., \& Tootell, R. B. (2003). Faces and objects in macaque cerebral cortex. Nature Neuroscience, 6, 989-995.

Vuilleumier, P. (2000). Faces call for attention: Evidence from patients with visual extinction. Neuropsychologia, 38, 693-700.

Vuilleumier, P., Armony, J. L., Driver, J., \& Dolan, R. J. (2003). Distinct spatial frequency sensitivities for processing faces and emotional expressions. Nature Neuroscience, 6, 624-631.

Winston, J. S., Vuilleumier, P., \& Dolan, R. J. (2003). Effects of low-spatial frequency components of fearful faces on fusiform cortex activity. Current Biology, 13, 1824-1829.
XU, Y. (in press). Revisiting the role of the fusiform face area in visual expertise. Cerebral Cortex.

YIN, R. K. (1969). Looking at upside-down faces. Journal of Experimental Psychology, 81, 141-145.

\section{NOTES}

1. However, it is worth noting that analyses in the left FFA for Experiment 1 suggested the same pattern of results as that in the right FFA. The following correlations were observed: behavioral car expertise with car - face activity for HSF, $.52(p=.15)$; behavioral car expertise with car - face activity for LSF, $77(p=.04)$; behavioral car expertise with car - face activity for the whole task, $.84(p=.01)$; behavioral car expertise with car - face activity for the part task, $.57(p=.12)$; car face activity for LSF and car - face activity for HSF, $.13(p=.813)$; car - face activity in the whole task and car - face activity for the part task, $.51(p=.305)$.

2. Cars and faces in Experiment 3 were shown on textured backgrounds (a single broadband background for all the objects, which contained both LSF and HSF components and, after filtering, left only LSF components in the LSF stimuli and vice versa). It is possible that the LSF content of the background impaired figure-ground segregation more than its HSF content did, which could account for the HSF behavioral advantage in Experiment 3, as compared with the other two experiments with LSF advantages.

(Manuscript received June 28, 2004; revision accepted for publication April 15, 2005.) 